\title{
Chondrosarcoma Metastasis to the Choroid
}

\author{
Jenna May Kim ${ }^{a}$ Amanda J. Wong ${ }^{a}$ Amanda J. Lu ${ }^{a}$ Renelle Pointdujour-Lima, b \\ ${ }^{a}$ Department of Ophthalmology and Visual Science, Yale New Haven Hospital, Yale School of Medicine, New Haven, \\ CT, USA; ${ }^{b}$ Ophthalmic Oncology, Smilow Cancer Center, Yale School of Medicine, New Haven, CT, USA
}

\section{Established Facts}

- Chondrosarcoma is a rare type of bony cancer.

- Uveal metastasis from bony cancers are extraordinarily rare and all pre-existing reports are of unilateral presentations.

\section{Novel Insights}

- Chondrosarcoma can present as uveal metastasis to both eyes simultaneously.

- Co-management of metastatic patients by ophthalmology and medical oncology is recommended.

\section{Keywords}

Chondrosarcoma - Choroidal metastasis - Choroidal tumor . Sarcoma

\begin{abstract}
We report a rare case of chondrosarcoma metastatic to the choroid. A 64-year-old male with a history of chondrosarcoma metastatic to the lungs and to the spine presented with blurred vision. A choroidal tumor was found. Fine-needle biopsy confirmed the histologic identity of the tumor as chondrosarcoma. Metastatic spread of chondrosarcoma to the eye is extremely rare. When present, lesions may grow rapidly, and systemic prognosis is poor. Co-management with medical oncology is of utmost importance. This is the third case of chondrosarcoma metastatic to the choroid in the literature and the first with bilateral involvement.
\end{abstract}

(c) 2019 S. Karger AG, Basel

\section{KARGER}

(c) 2019 S. Karger AG, Basel

E-Mail karger@karger.com

www.karger.com/oop

\section{Introduction}

Chondrosarcoma belongs to a group of rare tumors that arise from bony mesenchymal tissue. Unlike Ewing sarcoma and osteosarcoma, two other types of primary malignancies of the bone, chondrosarcoma is found in patients between the 4 th and 7 th decade of life and most often involves the scapula, ribs, sternum, or pelvis $[1,2]$. It is nonetheless a rare disease with an estimated incidence of 1 in 200,000 per year in the United States [3], and together with osteosarcoma and Ewing sarcoma make up less than $0.2 \%$ of all malignancies [4]. The most common sites of metastatic spread of chondrosarcoma are the lungs. To our knowledge, only 2 prior cases in the litera-

Jenna M. Kim and Amanda J. Wong contributed equally to the manuscript preparation. 

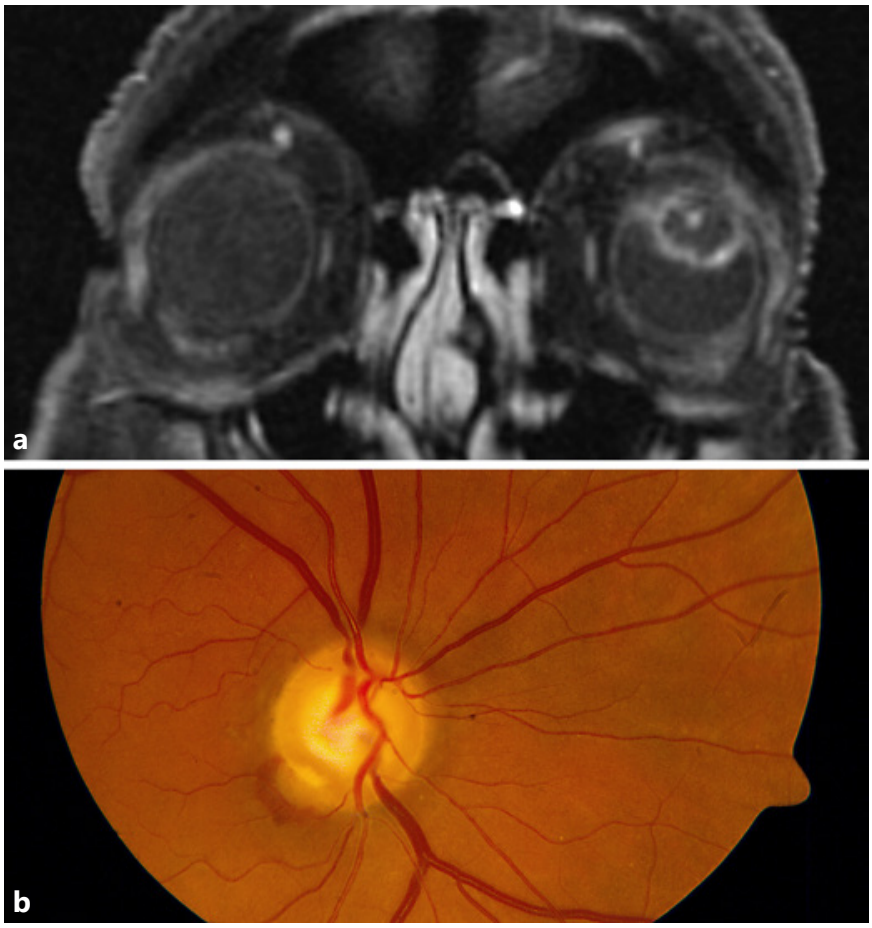

Fig. 1. a Magnetic resonance imaging of the orbits showing an obvious mass in the left globe superiorly. The lesion was measured to be $14 \times 10 \times 11 \mathrm{~mm}$ in size. b A small peripapillary lesion $\mathrm{OD}$ is visible with surrounding hemorrhage.

ture describe intraocular chondrosarcoma metastasis [5, 6], suggesting even greater scarcity of intraocular involvement of this rare malignancy. Herein, we describe a case of biopsy-proven chondrosarcoma metastatic to the choroid.

\section{Case Report}

A 64-year-old male with a history significant for chondrosarcoma metastatic to the lungs and to the spine presented to the emergency department with complaints of left-eye pain and decreased vision. He had been diagnosed with localized myxoid chondrosarcoma of the right rib 15 years before at an outside hospital and had undergone local tumor resection. Seven years after the initial diagnosis, he developed thoracic spine metastasis that required surgical resection, external beam radiation therapy, and chemotherapy. Lung metastasis was found 4 months before the ocular presentation, and he had undergone palliative external beam radiation therapy. Given his new ocular symptoms and extensive metastatic chondrosarcoma history, magnetic resonance imaging of the brain and orbits was obtained in the emergency department. The imaging showed an intraocular mass measuring $14 \times 10 \times 11 \mathrm{~mm}$ (Fig. 1a) in the left eye (OS).

Chondrosarcoma Metastatic to the Choroid
He was evaluated in the Ophthalmic Oncology Section at the Yale Cancer Center. On examination, his best-corrected visual acuity at distance was 20/25 in the right eye (OD) and 20/80 OS. His pupils were equal and reactive to light and without relative afferent pupillary defect. He demonstrated an inferior hemi-field deficit OS on confrontational visual fields. Anterior segment examination was unremarkable. Fundoscopic examination OD revealed a small peripapillary lesion with surrounding hemorrhages (Fig. 1b). More notably in OS, an amelanotic choroidal mass was seen; it measured $18 \mathrm{~mm}$ in basal diameter and $12.6 \mathrm{~mm}$ in thickness with an inferior serous retinal detachment (Fig. 2a). The optic disc was visible. Optical coherence tomography confirmed the extent of subretinal fluid. B-scan ultrasonography showed an echogenically heterogeneous mass. Options for management included observation, plaque brachytherapy, external beam radiation, or enucleation. The patient opted for observation in the setting of known lung metastasis.

The patient returned for an urgent visit 12 days later for intractable pain OS. The visual acuity OS had diminished to counting fingers at 1 foot. There was an increase in the size of the lesion OS to 20 $\mathrm{mm}$ in basal diameter and $15 \mathrm{~mm}$ in thickness. The optic disc was completely obscured by the mass (Fig. $2 \mathrm{a}-\mathrm{f}$ ). The patient underwent fine-needle aspiration biopsy of the choroidal lesion OS to confirm the diagnosis. At his postoperative visit, the patient reported resolution of pain OS. There was no change to the status of OD.

\section{Results}

Cytopathologic analysis of the aspirate demonstrated a metachromatic staining matrix with neoplastic chondrocytes consistent with metastatic chondrosarcoma to the choroid. The patient refused further evaluation or treatment. He was referred to medical oncology for systemic management but was lost to follow-up despite extensive efforts to reach him.

\section{Discussion/Conclusion}

Although metastatic disease is the most common cause of choroidal tumors $[7,8]$, bone sarcoma metastasis to the choroid is exceedingly rare. To our knowledge, there have been 9 published cases of intraocular metastases from bone sarcoma $[5,6,8-14]$, only 2 of which were from chondrosarcoma [5, 6]. In 1996, George and Zamber [5] described the first case of chondrosarcoma metastatic to the eye. The patient presented with a 3-day history of eye pain and decreased vision in one eye and eventually required enucleation for painful blind eye from end-stage mechanical glaucoma. The second case of chondrosarcoma metastatic to the choroid was published in 2012 [6], though the choroidal mass was never biopsied as the patient was lost to follow-up.

Ocul Oncol Pathol 2019;5:234-237 

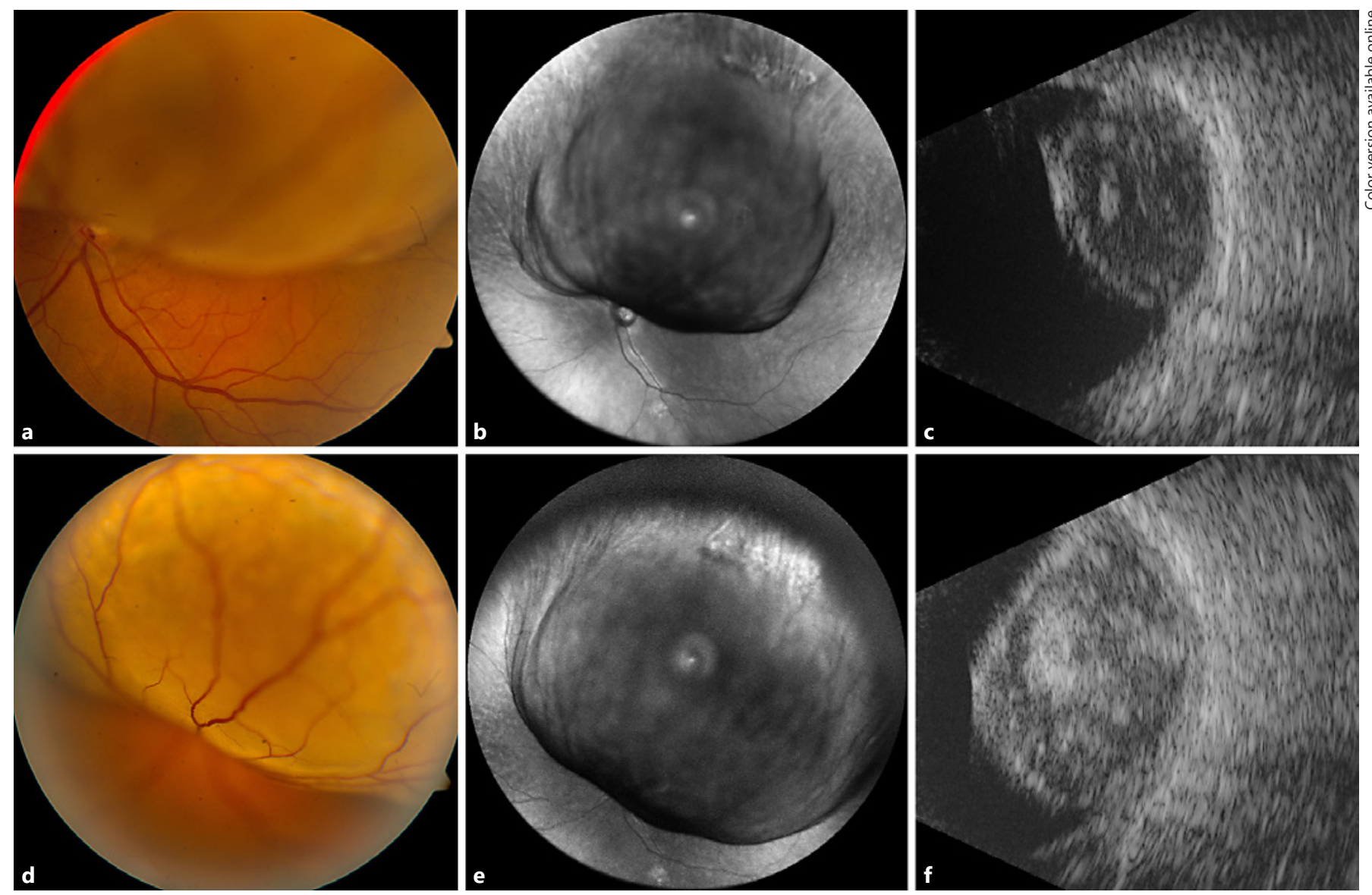

Fig. 2. Clinical images of the choroidal mass OS. a-c Initial presentation. $\mathbf{d - f}$ Subsequent images 12 days after the initial presentation. a, d Color fundus photograph OS with a superior choroidal mass, initially with the visibility of the optic nerve preserved (a) that is subsequently eclipsed by the mass (d). b, e Autofluorescence wide- angle image of the superior choroidal mass, initial view (b) and subsequent view (e). c, f B-scan ultrasonography of the mass on initial visit (c) versus the subsequent visit (f). Enlargement of the mass in all dimensions is appreciated in the initial images $(\mathbf{a}-\mathbf{c})$ compared to the subsequent images $(\mathbf{d}-\mathbf{f})$.
Several common themes are notable in the small cohort of patients with bone sarcoma metastatic to the eye reported in the literature. In 8 of these cases, there was a history of systemic metastasis from the primary malignancy. Ocular presentation was unilateral in all 8 reports that specified laterality. Of the 6 cases with known followup, all 6 patients died within 12 days to 2 years from the diagnosis of intraocular metastasis from complications of the cancer. The present case marks the third case in the literature of chondrosarcoma metastatic to the choroid and is the first known primary bone sarcoma case to present with bilateral choroidal metastasis.

Although definitive conclusions cannot be drawn from the existing small number of case reports, it may be reasonable to infer that choroidal metastasis from sarcoma portends a poor overall prognosis. Intraocular in- volvement correlates with the heavy burden of disease. For patients with a known history of sarcoma presenting with new ocular complaints, an expedient metastatic workup and close follow-up is recommended. Given the poor prognosis of patients with sarcomas metastatic to the eye, it is advised that these patients be co-managed by ophthalmology and medical oncology.

\section{Acknowledgement}

The authors thank the ophthalmic photographers Catherine Goodwin and Brenda Goss for the acquisition of the clinical images for this case. We are also grateful to Dr. Mario Sznol and his team for attempting to arrange medical oncology care for the patient. 


\section{Statement of Ethics}

The authors have no ethical conflicts to disclose. The patient provided written consent for the publication of his clinical images.

\section{Disclosure Statement}

The authors have no conflicts of interest to declare.

\section{Funding Sources}

The authors did not receive any funding.

\section{Author Contributions}

Amanda J. Wong wrote the first draft of the manuscript. Jenna M. Kim was involved in the management of the patient and major revisions of the manuscript. Amanda J. Lu contributed to the editing of the manuscript. Renelle Pointdujour-Lim was the primary ophthalmic oncologist managing the patient from presentation to biopsy and also contributed to the editing of the manuscript.

\section{References}

1 Mery B, Espenel S, Guy JB, Rancoule C, Vallard A, Aloy MT, et al. Biological aspects of chondrosarcoma: leaps and hurdles. Crit Rev Oncol Hematol. 2018 Jun;126:32-6.

2 Jacques C, Renema N, Lezot F, Ory B, Walkley CR, Grigoriadis AE, et al. Small animal models for the study of bone sarcoma pathogenesis:characteristics, therapeutic interests and limitations. J Bone Oncol. 2018 Feb;12:7-13.

3 Giuffrida AY, Burgueno JE, Koniaris LG, Gutierrez JC, Duncan R, Scully SP. Chondrosarcoma in the United States (1973 to 2003): an analysis of 2890 cases from the SEER database. J Bone Joint Surg Am. 2009 May;91(5): 1063-72.

4 Evola FR, Costarella L, Pavone V, Caff G, Cannavò L, Sessa A, et al. Biomarkers of Osteosarcoma, Chondrosarcoma, and Ewing Sarcoma. Front Pharmacol. 2017 Apr;8:150.
5 George DP, Zamber RW. Chondrosarcoma metastatic to the eye. Arch Ophthalmol. 1996 Mar;114(3):349-51.

6 Francés-Muñoz E, Gallego-Pinazo R, PardoLópez D, Diaz-Llopis M. Choroidal metastasis from chondrosarcoma. Graefes Arch Clin Exp Ophthalmol. 2012 Jun;250(6):949-51.

7 Shields CL, Shields JA, Gross NE, Schwartz GP, Lally SE. Survey of 520 eyes with uveal metastases. Ophthalmology. 1997 Aug;104 (8):1265-76.

8 Shields CL, Welch RJ, Malik K, Acaba-Berrocal LA, Selzer EB, Newman JH, et al. Uveal Metastasis: Clinical Features and Survival Outcome of 2214 Tumors in 1111 Patients Based on Primary Tumor Origin. Middle East Afr J Ophthalmol. 2018 Apr-Jun;25(2):81-90.

9 Green DM, Marinello MJ, Fisher J, Mindell ER, Zak TA, Allen JE, et al. Ewing's sarcoma of the scapula with metastases to the lung and eye. Am J Pediatr Hematol Oncol. 1986;8(2): $134-43$.
10 Gündüz K, Shields JA, Shields CL, De Potter $\mathrm{P}$, Wayner MJ. Ewing sarcoma metastatic to the iris. Am J Ophthalmol. 1997 Oct;124(4): $550-2$.

11 Jampol LM, Cottle E, Fischer DS, Albert DM. Metastasis of Ewing's sarcoma to the choroid. Arch Ophthalmol. 1973 Mar;89(3):207-9.

12 Saito Y, Matsuzaki A, Suminoe A, Koga Y, Kurata H, Oda Y, et al. Congenital Ewing sarcoma in retroperitoneum with multiple metastases. Pediatr Blood Cancer. 2008 Nov; 51(5):698-701.

13 Less VT. A case of meatastatic osteosarcoma in the choroid. Br J Ophthalmol. 1947 Dec; 31(12):713-6.

14 Spaulding AG, Woodfin MC Jr. Osteogenic sarcoma metastatic to the choroid. Arch Ophthalmol. 1968 Jul;80(1):84-6. 